\title{
Study and Analysis Real-Time Methods of Tracking Objects on GPS and Mobile Telephone Towers Stations
}

\author{
FIRAS SHA WKAT HAMID \\ Electronic Techniques Department / Technical Institute Mosul / Northern Technical University \\ dr.firas.hamid@ntu.edu.iq
}

\begin{abstract}
GPS (Global Positioning System) has been used to track living beings and devices. It is a vital tool and indispensable over the last few years. Different types of applications including military and civil applications. In this work, a review of the modern application areas and a discussion of the benefits of GPS tracking are given. The software architecture of several tracking systems to position and track a lost child. An analysis of monitoring and tracking technologies are studied in this work. A comparison of different techniques is given. In this technology, navigation of the user (mother) to the destination (child) is implemented and the signal is analyzed. Real simulation of GPS receiver which enables the additional control of the user to the destination by using also GSM towers stations. The angle, position and distance are checked to know the exact position. The error signal has been analyzed for the system for more accurate results. So the new GPS system gives more accurate and fast results. This paper also reviews some of the new applications and discusses the benefits and issues of GPS tracking. It then presents a software architecture of a general tracking system to locate lost objects.
\end{abstract}

Keywords: GPS: Global Positioning System, RSS: Radio Signal Strength, AOA: Angle Of Arrival, TOA: Time Of Arrival, TDOA: Time Difference Of Arrival

\section{Introduction about study and analyzing ways of tracking moving objects}

The scope of this research work is to develop an application for tracking moving objects using simulation technique. Tracking Process is a very important process that is necessary in order to secure location information about a specific body whether this person is a human being as a child in a garden as in this project (or monitoring a mentally disturbed person, etc.) or a car as a surveillance of a responsible person, or an animal as a surveillance of pampered dogs in Western countries and fear of loss. Monitoring is an inverted process of positioning. Positioning is a very important issue and is part of a widespread science called navigation, which can be defined as the science that studies the determination of the beam state of moving or stationary objects. The state vector is the beam that houses the body (Three-dimensional) and vehicle speed and acceleration, etc.

The positioning process is largely related to the work environment in which the location of objects is required, often called the Coordinate System. On the ground, there are many orthodontic sentences, most notably the Cartesian coordinates, also known as the Earth Center Fixed Earth (ECEF), where the center of the earth is the center of this sentence and its axes are connected to the Equator and Greenwich lines. Also known as regular earth coordinates, any point of the earth's surface can be considered as the center of this sentence and its axes are chosen by default according to the available environment [1]. 
The main difference between the monitoring process and the positioning process is that the latter requires a number of transmitters and a single receiver to locate it. In the monitoring process, a single transmitter must be located and a number of receptors perform this task using a clustered algorithm that performs the calculation. It should be noted here that both processes use the same algorithms and the same measurements to achieve their purpose and the methods of observation that are used in practice in determining the location.

\section{Radio signal strength RSS}

Positioning using a signal is a very common method of positioning that uses a known mathematical model to dampen path loss with distance. Since the signal capacity measurements are provided by predicting the distance between the Tx and the Rx, the transmitter will be clearly located on the perimeter of the center of the receiving receiver. With two or more receptors, the location of the transmitter can be determined and will be the intersection point. However, Path Loss and Multipath Fading The accuracy of this method is modest, especially in densely populated environments. The distance error is significant when using the famous signal propagation models studied in these urban environments, which adversely affects the accuracy of the location [2].

In general, it is known that the receiving capacity is given in the following relation: $P_{r}(t)=P_{t}(t)-$ Losses

Where as, $P_{r}(t)$ receiving power, $P_{t}(t)$ the power sent from the transmitter and Loses: lost signal.

The study of propagation loss is related to multiple parameters and is closely related to the transmitter and receiver working environment. There are famous sports models and models known to express the models of the spread of the signal, including but not limited to [2], [3]:

\subsection{Free Space Propagation Model}

This model is based on the principle of signal delay as the distance between the Tx and the Rx. The loss of the path in this model is given when taking antenna gain into account with the following relation $[2,3]$ :

$$
\text { Losses }=\frac{(4 \pi)^{2} \cdot d^{2}}{\lambda^{2}}
$$

Where $\mathrm{d}$ : is the distance between sender and receiver, $f:$ is frequency $(\mathrm{MHz})$.

However, this model suffers from many errors because it does not take into account the conditions surrounding the spread of obstacles and buildings and this model is one of the simplest and easiest models used.

\subsection{Longley-Rice model}

This model is widely used in wireless communications and is a pilot model based on maps called Longley Rice Maps showing the low radio signal resulting from the shapes and nature of regions and climate. This model operates in the bandwidth range of $20 \mathrm{MHz}$ to $20 \mathrm{GHz}$ and takes into account the following [2], [3]:

- Atmospheric absorption.

- Precipitation resulting from precipitation.

- The heavenly noise.

- Roughness and roughness of the studied environment.

- Refraction of acute endings.

- Forget the scabies. 
- Dimmed signal with long distances.

It does not take into account the impact of ionospheric weather, and this model was adopted by the Commission Federal Communications. This model is not based on a fixed mathematical formula, but rather on the collection of the above-mentioned impedances and adding them to the value of the path loss resulting from the spread of free space.

\subsection{HATA / OKUMURA model}

This model is also experimental and has been applied in the study of the propagation of waves of cellular systems in different circumstances of these environments and works in the field of frequency from 150 to $1500 \mathrm{MHz}$. This model is determined by distance, frequency and height of antennas and place (cities, suburbs, countryside, .....). This model was based on a densely populated urban environment and a correction factor was used to disseminate this formula to suburban and rural areas. HATA and OKUMURA did not take into consideration the obstacles between the transmitter and the receiver (hills, mountains, buildings ...), from the principle that they considered that transmitters were usually placed on high hills and hills. The loss of path in this model for urban or urban environments is given in relation to [2], [3]:

$$
\operatorname{Losses}(\mathrm{dB})=\mathrm{A}+\mathrm{B} \cdot \log (\mathrm{d})
$$

Where (A) is the constant loss depends on the frequency of the signal and according to the following relationship:

$$
A=69.55+26.16 \log (f)-13.82 \log \left(h_{b}\right)-a\left(h_{m}\right)
$$

Whereas:

$h_{b} \quad:$ Tx Antenna Height (m), $h_{m}:$ Rx Antenna Height (m)

$a\left(h_{m}\right)$ : The correction factor in the small and medium cities is estimated in decibels and is given by the relationship:

$a\left(h_{m}\right)=[1.11 \log (f)-0.7] h_{m}-[1.56 \log (f)-0.8]$

The path loss model in this model for suburban areas without considering the noise factor:

$$
\text { Losses }(d B)=\text { Losses }(d B)_{U r b a n}-2\left[\operatorname{tg}\left(\frac{f}{28}\right)\right]^{2}-5.4
$$

And taking the Correction Factor in account to become the relationship:

$$
\text { Losses }(d B)=\operatorname{Losses}(d B)_{\text {Urban }}-2\left[\operatorname{tg}\left(\frac{f}{28}\right)\right]^{2}
$$

\subsection{Model COST-231 HATA}

It is an expansion and expansion of HATA / OKUMURA for PCS (personal communication system) operating from $1800 \mathrm{M}$ to $2000 \mathrm{MHz}$. The COST, based on the HATA / OKUMURA model, developed a model called COST - 231, It relies mainly on four key parameters to express loss of path [2], [3]:

- Frequency.

- The height of the receiver antenna.

- High transmitter antenna.

- Distance between transmitter and receiver.

- Loss of track in urban areas is given in relation to: 


$$
\text { Losses }(d B)=46.33+33.9 \log (f)-13.82 \log \left(h_{b}\right)-a\left(h_{m}\right)+Q
$$

Where as:

$Q=\left[44.9-6.55 \log \left(h_{b}\right)\right] \log (d)$

$$
\mathrm{a}\left(\mathrm{h}_{\mathrm{m}}\right)=[1.11 \log (\mathrm{f})-0.7] \mathrm{h}_{\mathrm{m}}-[1.56 \log (\mathrm{f})-0.8]
$$

In the suburbs and highways, the bug is based on the same relationship as the HATA / OKUMURA model.

\section{The Steric Field Technique}

This method of location determination depends on the use of a multi-beam antenna receiver (or multiple antennas) for each beam or antenna (if there are a number of antennas) covering a specific space segment so that the entire space surrounding the receiver is covered [4], [5]. In order to determine the location of an object that sends a specific signal, it is necessary to have at least two receiver receivers of the above type, each of which determines the radiation beam receiving the transmitter signal and with a link between both receptors the approximate location of the transmitter can be determined as shown in figure 1.

However, this method suffers from the problem of the lack of precise identification as shown in the figure where the location of the sender is located in the form of a small space segment resulting from the intersection of radiated beams received in both receivers, and therefore the accuracy here linked to the beam beams of the receivers, The more accurate the transmitter's location, the more complex the receiver antennas will be and the greater the cost of these antennas.

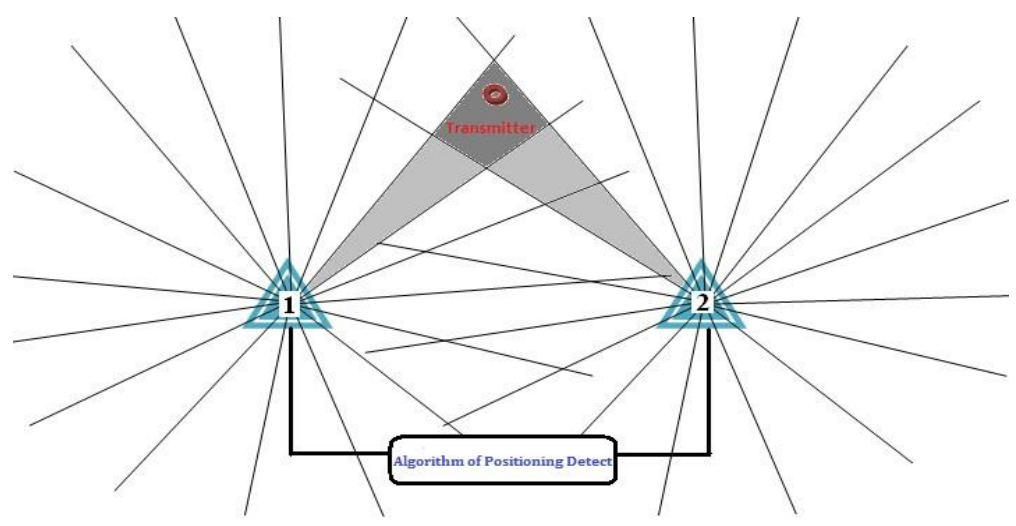

Fig. 1. Determination of the location of a transmitter by the space method.

\section{Method of access angle technique AOA (Angle Of Arrival)}

This method is very effective and practically depends on the existence of the wireless pointer (Guide) device (Direction Finder) that measures the angle of access (Angle Of Arrival) to signal the sender to be located. The determination of the access angle is for a specific reference direction and is often the North Direction, as shown in figure 2 [4], [5], [6]. 


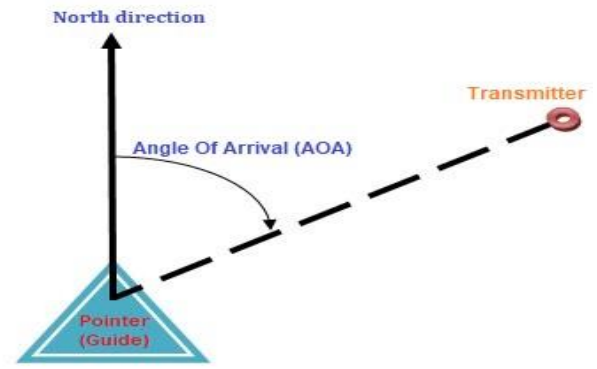

Fig. 2. Determination the Angle Of Arrival for the transmitter signal.

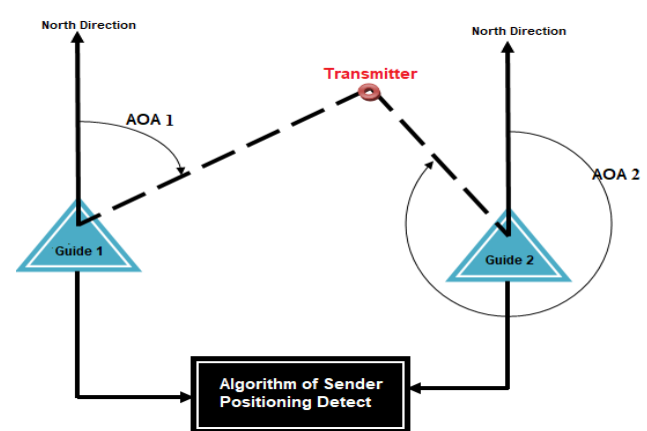

Fig. 3. Positioning locate using Angle of arrival Algorithm.

By measuring the angle mentioned, the receiver would have located the sender on a halfstraight start at the future. In order to determine the place on this rectum, we also need another pointer (Guide), as in the previous method, and thus define another half-straight, and the transmitter is at the intersection of the rectum as shown in Figure 3. This method is very accurate and simple in arithmetic, but the main problem is the complexity of angular measurement as it depends on the use of rotary antennas with a special radiation pattern and large dimensions depending on the frequency used in the transmitter.

\section{Time of Arrival (TOA) method}

This method is similar to the previous method, but the only difference is in the measured parameter. This method depends on the measurement of the time delay of the signal propagation from the transmitter to the receiver. Signals of both transmitter and receiver in order to be able to measure delay time. By measuring the delay time we can calculate the distance between the transmitter and the receiver depending on the known relationship [4], [5], [6]:

$$
D=c . \Delta t
$$

To set the distance to the transmitter we can, as in the case of measuring the ability to know the place of the sender on. The circumference of the center circle its future as shown in figure 4.

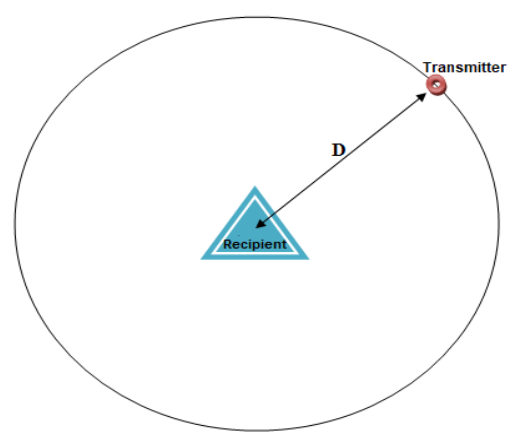

Fig. 4. Determining the distance to the sender. 
By measuring the distance between the $\mathrm{Tx}$ and the $\mathrm{Rx}$, the receiver will locate the transmitter on the perimeter of the circle as shown in figure 4. In order to locate the transmitter on this circuit, we need another receiver and thus define another circle, as shown in figure 5. This is one of the most accurate and simple methods of calculation and measurement, but the main problem is the need for synchronization between signals transmitter and receiver, this problem is not easy as there are many techniques of synchronization can be studied in other research.

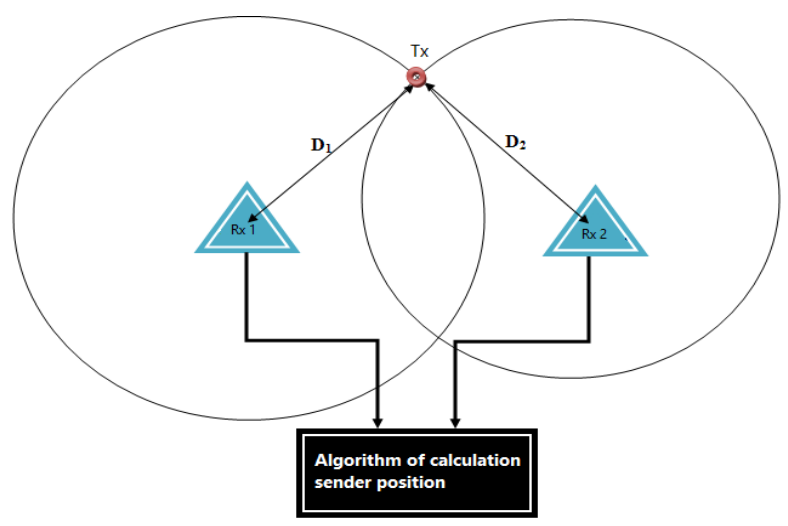

Fig. 5. location determined by measuring Time of Arrival (TOA)

\section{TDOA technique (Time Difference Of Arrival)}

This method is close to the previous method (measured in the access time) but in this way, there will be no interest in the signal arrival time from the transmitter to the receiver but the difference between the delay times between two signals Time Difference Of Arrival. Therefore, in order to measure this parameter, we need two receivers as shown in figure 6 [4], $[5],[6]$.
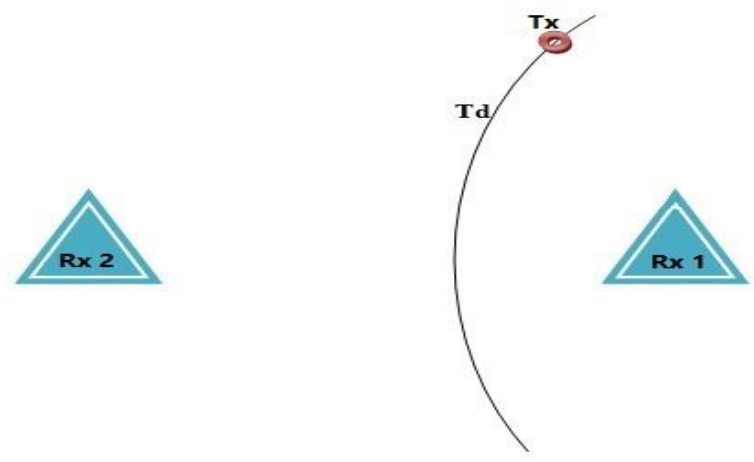

Fig. 6. Measuring the Time Difference Of Arrival

Note that in order to measure the difference in access times for transmitter signals between two receptors we determine the location of this transmitter is on a plus (Hyperbolic). In order to locate the transmitter at any of these points, we need another measurement of the difference in access times and this requires the presence of the other two transmitters or at least another transmitter as shown in figure 7. This method is also a fine way because it presents common 
errors in the signaling path between the transmitter and receiver, but it is very complicated and very expensive as it requires more receptors than the previous methods.

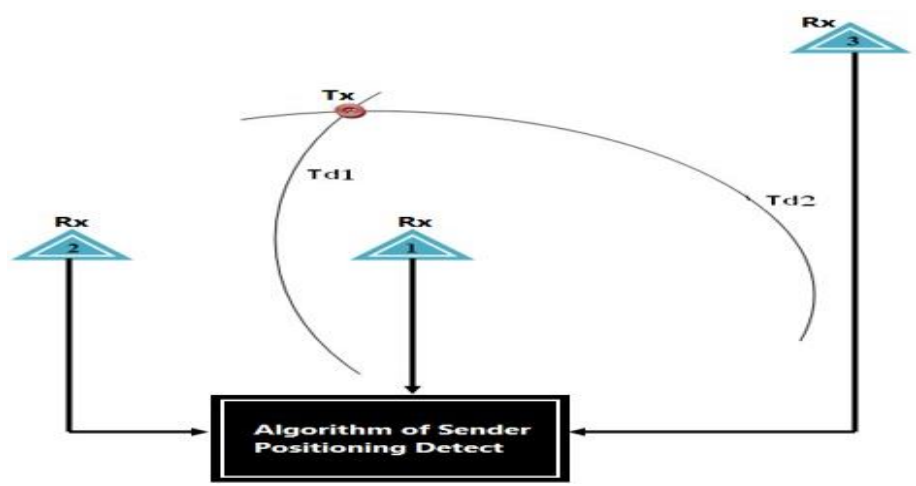

Fig. 7. Determination of the sender's location using the TDOA technique.

\section{Monitoring technique using the GPS system}

Recently, the Global Positioning System (GPS) has emerged as a modern system capable of accurately positioning objects. This system is able to calculate and determine the future of the ways to deal with a number of satellites that move in space on specific orbits and known. The GPS receiver is called the GPS Tracker. The GPS receiver is then accurately measured from the GPS receiver and the location information is transmitted, and therefore there is a single receiver. As an example (Vehicle Control), accurate and instantaneous information about the location and movement of the desired object can be obtained. This method is considered one of the best methods because of its simplicity and reasonable cost as well as accuracy in positioning, which is the result of the accuracy of the future of GPS [4], [7], [8].

\section{Analysis of control methods}

Through the previous studies described in this paper, we can build an analytical study and compare the different methods of observing an object, and always the basic criterion in comparison is the accuracy of the location because this criterion achieves the purpose of this technique. Table (1) shows a simple comparison between the methods mentioned above, where we note that each method advantages and disadvantages and therefore the choice of the appropriate method is due to the quality of the body required to monitor and the importance of locating the exact accuracy and the cost allocated to such a project.

\section{Analysis of the capacity technology algorithm:}

Two methods of study were chosen. The first is the technique of the ability of the wireless signal according to the propagation model of the signal in the free space, and the second is the method of using the GPS receiver [1,8].

\subsection{Technical modeling of signal capacity according to the free space model}

Positioning using a signal is a very common method of positioning that uses a known mathematical model to dampen path loss with distance. Since the signal capacity measurements are provided by predicting the distance between the transmitter and the receiver, the transmitter will be clearly located on the perimeter of the center of the receiving receiver. With two or more 
receptors, the location of the transmitter can be determined and will be the intersection point. However, Path Loss and Multipath Fading The accuracy of this method is modest, especially in densely populated environments. The distance error is significant when using the famous signal propagation models studied in these urban environments, which adversely affects the accuracy of the location.

Table 1. Comparison of methods of monitoring an object

\begin{tabular}{|c|c|c|c|}
\hline Negativity & Positivity & The way & \\
\hline Modest accuracy & $\begin{array}{l}\text { Simple calculations and simple } \\
\text { processing do not require } \\
\text { synchronization }\end{array}$ & Capacity & 1 \\
\hline Multi-cost antennas & $\begin{array}{l}\text { Simple calculations and simple } \\
\text { processing do not require } \\
\text { synchronization }\end{array}$ & Stereotypical & 2 \\
\hline $\begin{array}{l}\text { Methods of measurement } \\
\text { of complex direction }\end{array}$ & $\begin{array}{l}\text { Simple calculations and simple } \\
\text { processing do not require } \\
\text { synchronization }\end{array}$ & Access angle & 3 \\
\hline need to synchronize & $\begin{array}{l}\text { Simple calculations and simple } \\
\text { processing - high accuracy }\end{array}$ & Time of arrival & 4 \\
\hline $\begin{array}{c}\text { Need to synchronize - great } \\
\text { cost }\end{array}$ & High accuracy & Time difference & 5 \\
\hline- & $\begin{array}{l}\text { High accuracy - simplicity in } \\
\text { implementation }\end{array}$ & GPS method & 6 \\
\hline
\end{tabular}

In general, it is known that the receiving capacity is given in the following relation:

$$
P_{r}(t)=P_{t}(t)-\text { Losses }
$$

Where as:

$\mathrm{P}_{\mathrm{r}}(\mathrm{t})$ Receiving power.

$\mathrm{P}_{\mathrm{t}}(\mathrm{t})$ The power sent from the transmitter.

Losses Propagation Loss

The study of propagation loss is related to multiple parameters and is closely related to the transmitter and receiver working environment. There are famous sports models and models known to express the signal propagation models:

- Free Space Propagation Model.

- Longley-Rice model.

- HATA / OKUMURA model.

- Cost-231 HATA Model.

This model is based on the principle of signal delay as the distance between the transmitter and the receiver. The loss of the path in this model is given when taking antenna gain into account with the following relation: 


$$
\text { Losses }=\frac{(4 \pi)^{2} \cdot d^{2}}{\lambda^{2}}
$$

Where $d$ is the distance and $f$ is the frequency in MHz.

Figure 8 shows the change of losses as a frequency dimension at a different distance between transmitter and receiver. We observe that the loss increases with increasing frequency and increases with increasing distance between transmitter and receiver. However, this model (signal capacity) suffers from many errors because it does not take into account the conditions surrounding the spread of obstacles and buildings and is considered this model is one of the simplest and easiest models used.

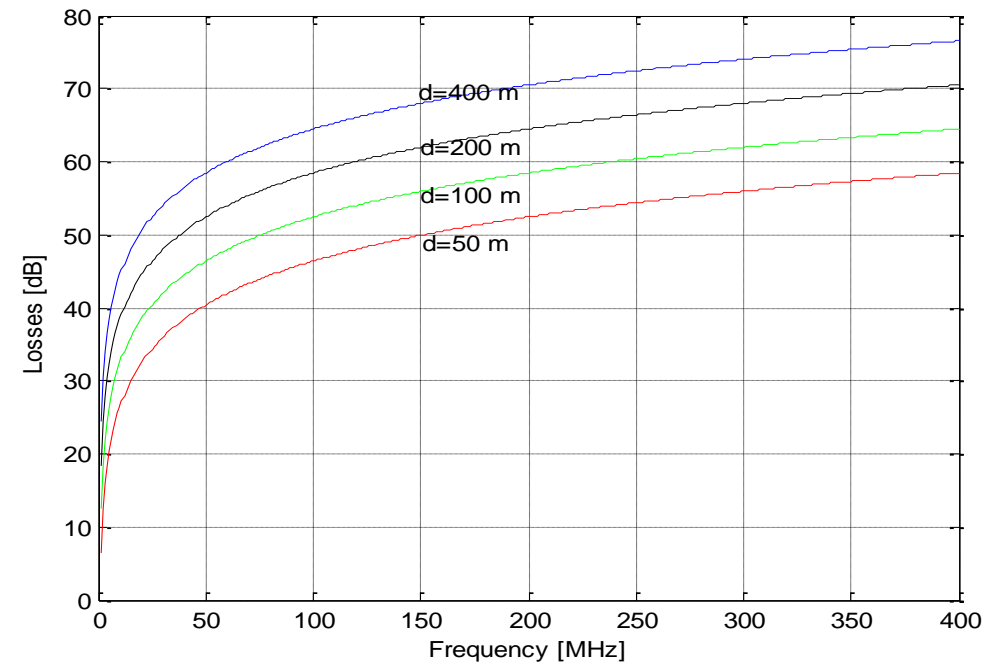

Fig. 8. Changes of losses as a frequency variable at different values of the distance between transmitter and receiver.

The distance measurement error is also increased by increasing the distance between the Tx and the $\mathrm{Rx}$ as shown in figure 9 , so it is preferable to use this method in the case of long distances. 


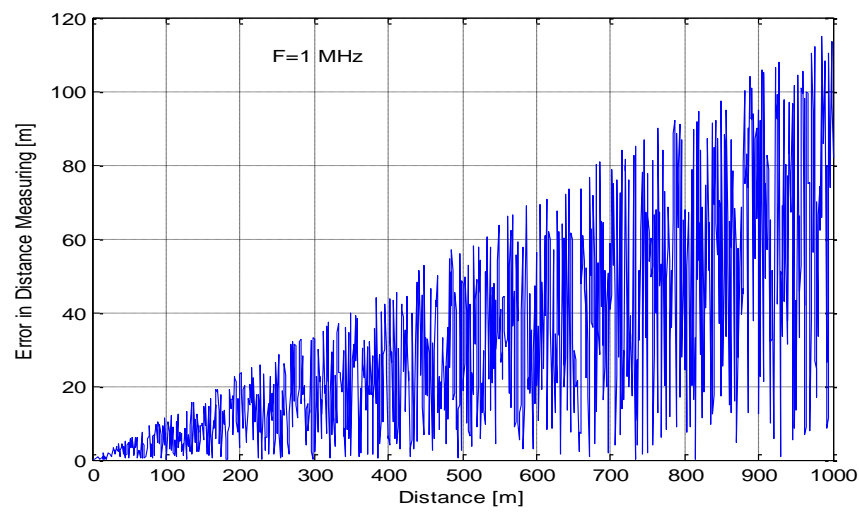

Fig. 9. change the distance measurement error with the changing of the distance between the transmitter and receiver.

\subsection{Algorithm finding the transmitter location}

The algorithm for determining coordinates in this method is based on measured distance information from distance measuring devices and send it to the computer of navigation that it results will send it to the display and notification devices, all these steps are defined by the monitoring system [7]. The method of determining coordinates based on distance measurement is one of the easiest and oldest methods of determining the location. The method of determining the coordinates of a sender's position is based on measuring the range " $\mathrm{d}$ " to a point of reference. Its ease is due to the ease of measuring distance. The transmitter is located at an unknown point with coordinates $(\mathrm{x}, \mathrm{y})$ to be determined. The range measurer measures the range from transmitter to receiver. The measured distance of this parameter is " $\mathrm{d}$ ". The value of this parameter means that the geometry of the transmitter's location is radius " $d$ " and its center of measurer itself. This circle is the positioning line for distance measurer. Figure 10 shows the location of the measurer and the transmitter's positioning line [9], [10], [11].

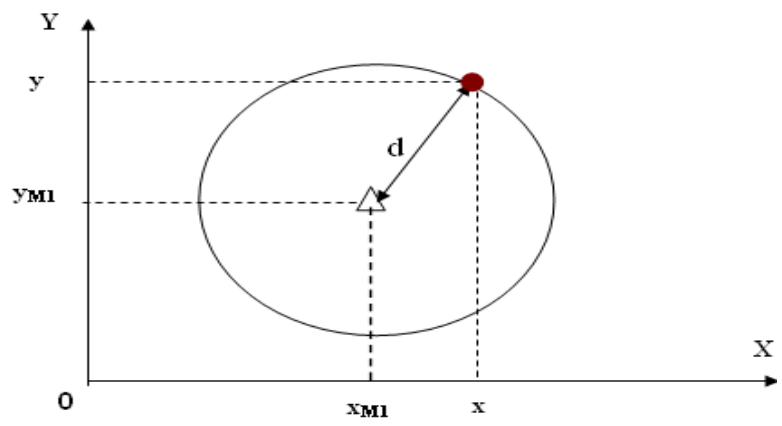

Fig. 10. The expected line of the sender's presence.

The analytical term of the dimension (two-dimensional) as a follower of the Cartesian coordinates of the transmitter and the measurer is a straight equation has the form: 


$$
d(x, y)=\sqrt{\left(x-x_{M}\right)^{2}+\left(y-y_{M}\right)^{2}}
$$

The task required here is to calculate the coordinates of the sender $\mathrm{x}, \mathrm{y}$. Returning to the last equation, we observe that " $\mathrm{d}$ " is a measured and knowing value and ym $\& \mathrm{xm}$ are a reference value and must be known and stored within the computer. Thus, this equation contains unknown (coordinate values) and this equation cannot determine the coordinates $\mathrm{x}, \mathrm{y}$. In order to find the required solution, it is necessary to have another navigational equation and this requires the presence of another measurer as in figure 11. Determination of the coordinates, in this case, is based on the knowledge of the measured values "d1", "d2" and the coordinates that previously given for measurer xm1, ym1, xm2, ym2.

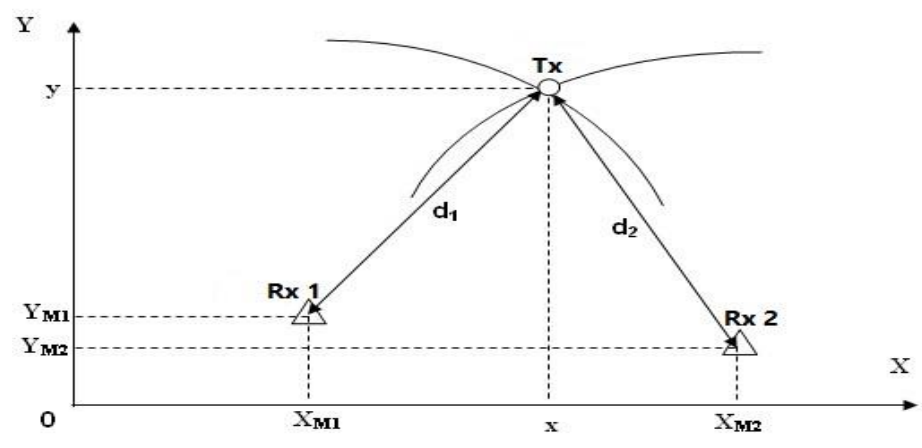

Fig. 11. Determines the position of a transmitter using distance measurer

So in order to resolve this case it should be known to us:

- Measured values of distances to sender $\mathrm{dm} 1, \mathrm{dm} 2$.

- Coordinates Measurer $x_{M 1}, y_{M 1}, x_{M 2}, y_{M 2}$.

The required that will specified here:

-Sender coordinates: figure 12 illustrates this situation.

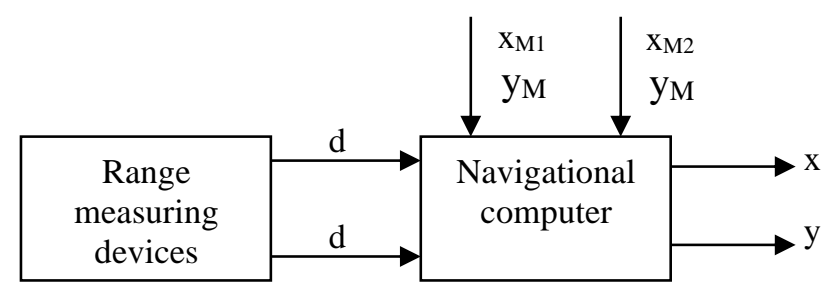

Fig. 12. Scheme for calculating a sender location in somewhere 
In order to find anonymous $\mathrm{x}$ and $\mathrm{y}$, we must solve a sentence consisting of two non-linear algebraic formulas. Nonlinear equations can be solved directly as follows :

$$
\begin{aligned}
& d_{1}(x, y)=\sqrt{\left(x-x_{M 1}\right)^{2}+\left(y-y_{M 1}\right)^{2}} \\
& d_{2}(x, y)=\sqrt{\left(x-x_{M 2}\right)^{2}+\left(y-y_{M 2}\right)^{2}}
\end{aligned}
$$

The relationship result becomes a second class for a variable $y$ and solve them will determine the value of $y$-coordinate and then determine the value of the $\mathrm{x}$-coordinate.

\subsection{Calculate the location of the transmitter}

Let us now assume that we have a monitoring system in place in a park. This system consists of two stations located according to figure 13. The first station is located in the coordinates $(x=350 m, y=50 m)$, while the second station is located at the point $(x=70 m, y=400 m)$. Both terminals are connected to the mainframe of the system by a standard cable, wireless card or other connection. Assuming that there is a lost child in this observation garden and that this child carries a transmitter sent at a known frequency.

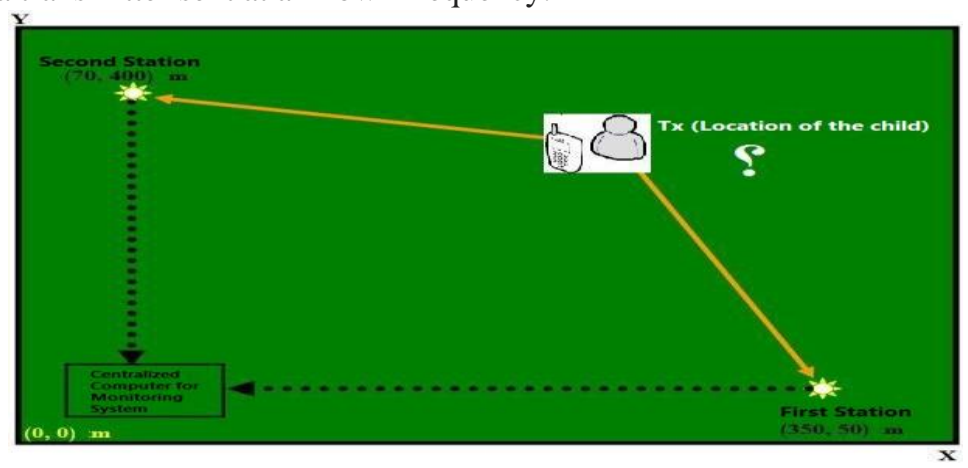

Fig. 13. A lost child in a garden and wants to locate a position.

Let us consider at the beginning of the study the absence of any error in measuring the distance, assuming that the measured values of the distance from the first stations and the second to the sender of the child are respectively:

$$
d_{1}=294.7 m d_{1}=215 m
$$

Once measured the distance will be sent to the computer system in order to calculate the place of the child according to the equations (13) and (14). And from it will get the equation of the second degree, and solve it we get the solution where:

$$
\mathrm{Y}=335 \mathrm{~m}, \quad \mathrm{x}=275 \mathrm{~m}
$$

Note from figure 13 that this value is accurate and true because measurement errors are not taken into account. If we consider the errors, according to Figure 9, the value of the two measured distances of the receivers respectively are: 


$$
d_{1} \approx 294.7 \pm 20=314 m d_{1} \approx 215 \pm 20=195 m
$$

With the same solution, we will note that there is an error in determining coordinates $\mathrm{X}$ and $\mathrm{Y}$.

\subsection{Positioning in GPS receivers}

The (GPS) Global Positioning Satellite System helps us for providing details regarding the position and time information to the user. The principle of positioning in GPS depends on the satellite transmitting radio signals continuously, any future receiving these signals and measure the range to the satellite. By measuring the range to at least three satellites, the receiver can determine its coordinates $(\mathrm{x}, \mathrm{y}, \mathrm{z})$. In order to obtain high accuracy in positioning using satellite navigational systems, very accurate hours are used in satellites. These atomic clocks and accuracy are greater than a second. The precision difference between the lunar clock and the receiver clock results in a presence [1], [12]. The false range sensor measures this range from the receiver to the satellite. The measured distance of this parameter is PR The value of this parameter means that the geometry of the receiver's future location is a PR-centered sphere, centered on the satellite. This ball is the level of position for approximate distance measurements. The analytical term of the approximate distance as a follower of the Cartesian coordinates of the receiver and the satellite is a straight equation and has the form:

$$
P R_{1}=R_{1}+\delta R=\sqrt{\left(x-x_{M 1}\right)^{2}+\left(y-y_{M 1}\right)^{2}+\left(z-z_{M 1}\right)^{2}}+\delta R
$$

The last equation is called the navigational equation for distance measures. With four satellites, the question becomes the solution for four equations. What is required now is to find the place of the (receiver coordinates) $\mathrm{z}, \mathrm{y}, \mathrm{x}$ based on the knowledge of measured values of distances to satellites PR1, PR2, PR3, PR4 and know the coordinates of reference points of these satellites $\mathrm{x}_{\mathrm{Mi}}, \mathrm{y}_{\mathrm{Mi}}, \mathrm{z}_{\mathrm{Mi}}$ as well as the real distance between the satellite and receiver $\left(\mathrm{R}_{1}\right)$ also the distance error caused by synchronization bands $(\delta \mathrm{R})$. The process of finding the coordinates of the receiver is combined by solving the nonlinear equations set out in relationship (15) for four equations. The solution of these nonlinear equations directly is accompanied by great difficulties. In order to simplify the process of solving the total of nonlinear algebraic equations, we convert them into linear and then perform the calculations of the repetitive [9], [13].

\section{Modeling a child monitor in a garden using GPS receivers:}

This method is easy and simple to implement where we need to implement the receptor of GPS with a wireless network card carried by the child to continue to move wherever he goes. The GPS receiver determines the coordinates of the child and sends location information via the wireless card to any receiver. Then it is possible for those who want to monitor the movement of the child to receive information place and see where this child's place accurately. figure 14 illustrates this technique. The time of sending the message from the satellite and receiving the message by the GPS receiver is compared. This time difference is used for calculating the distance between the receiver and satellite can be calculated, it calculates the distance from three satellites, therefore, there are minimum three satellites required to get any reading in a GPS. This process is also known as 2D-Position Fix. The most accurate reading can be obtained by using four satellites and it is called 3D-Position Fix. A 3D-Position Fix can even help determine the height above the earth's surface. 


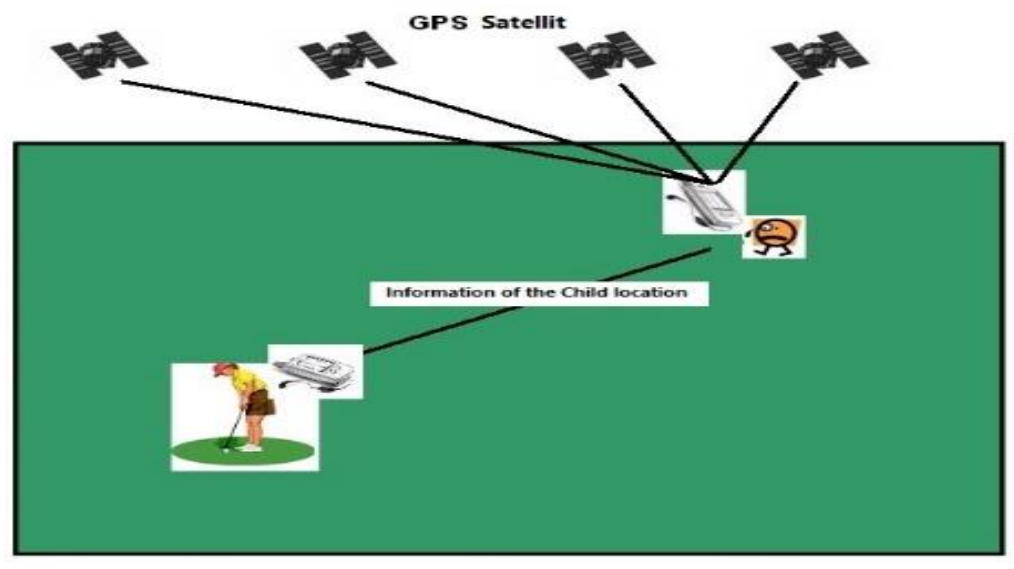

Fig. 14. Modeling the process of monitoring a child in a garden.

To calculate the errors, complete modeling was performed to determine the coordinates where the point of four satellites was assigned to transmit signals and the transmitter measured the distance and the coordinates. Figure 15 shows error values when moving on the $\mathrm{X}$ axis. Figure 16 shows error values when moving on the $\mathrm{Y}$ axis. We note that the error is averaged within 8 meters. The errors do not take into account multiple error Track (Multipath).

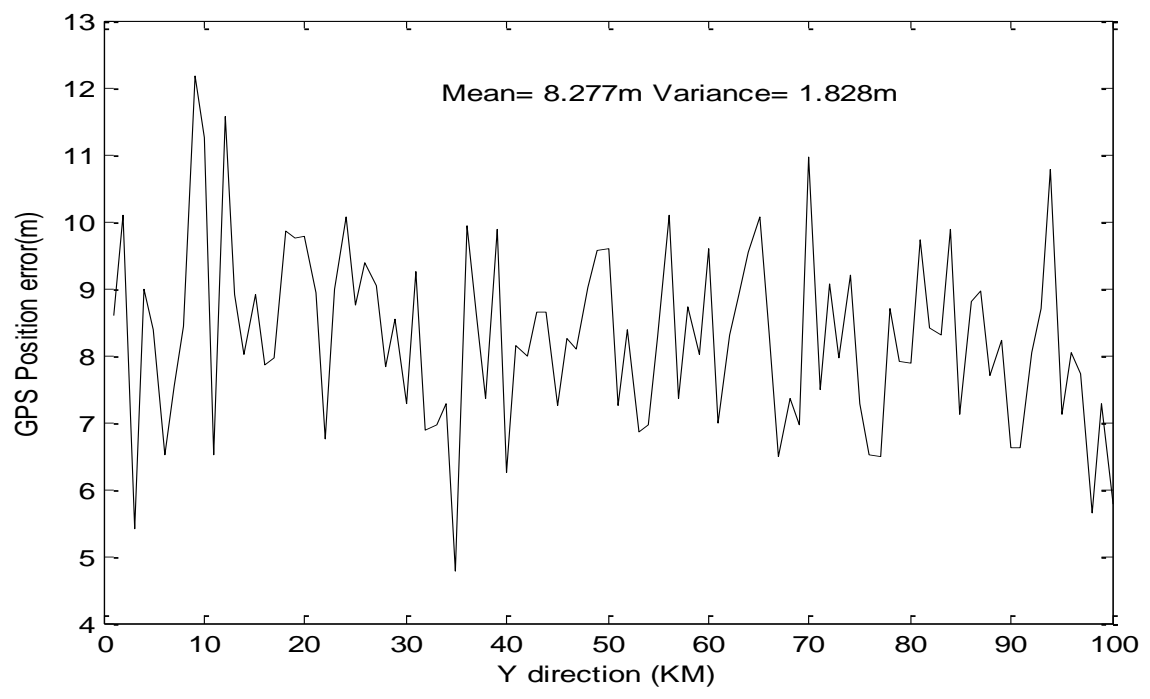

Fig. 15. GPS receiver error values when moving on the $X$ axis. 


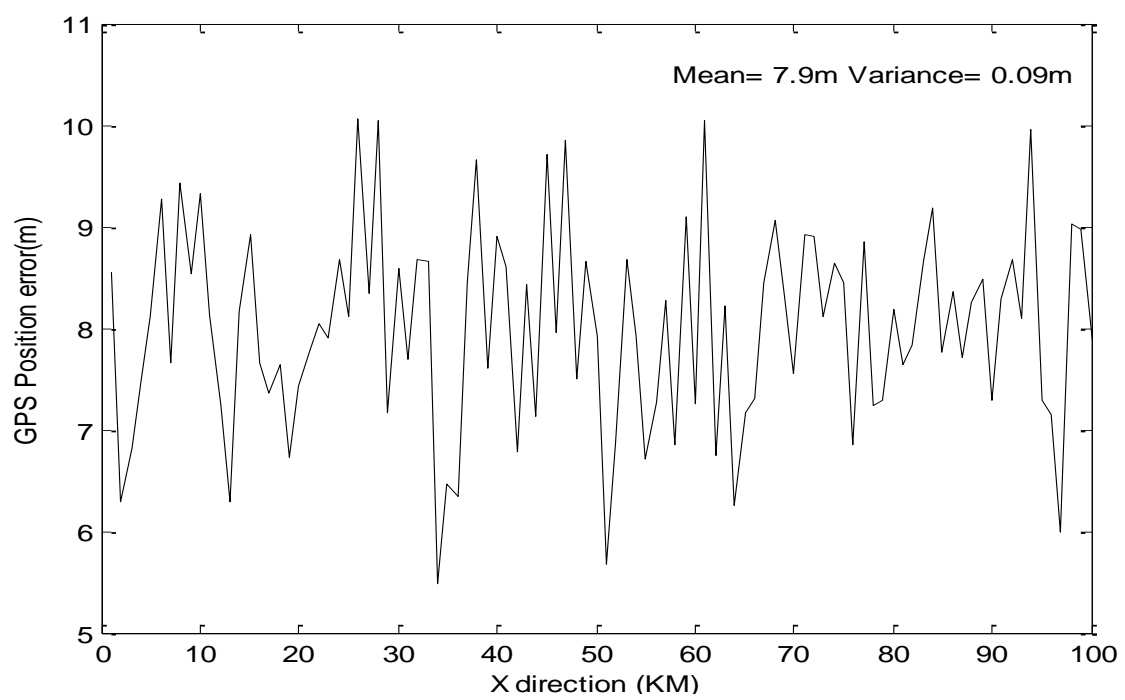

Fig. 16. GPS receiver error values when moving on the $Y$ axis.

\section{CONCLUSION}

The increase in the use of Mobile phones is targeting automation in every field of the world. Some modern application areas of GPS monitoring and tracking systems are discussed in this work. A mobile framework was proposed which implements a general tracking system. The system determines the position of a mobile device with or without GPS. So the system can be used to locate a lost or stolen child. It also can be used to locate valuables if they are equipped with the device. It is also helpful to locate people suffering from dementia or mental disorder. The proposed child monitoring system was studied and analyzed for a possible method of observation. The proposed system is much better and more efficient than the previous method and much easier to use and faster for a wide area such as a big park. By using this software, we can find out optimal routes when using a car or any transfer way for the journey and tracking of those routes even without the necessity of using GPS just using Mobile Telephone Towers Stations.

\section{References}

[1] Mao, A., Harrison C., Dixon, T.: Noise In GPS Coordinate Time Series. Journal of Geophysical Research, Vol. 104, No B2, Pages 2797-2816, February, 10, 1999.

[2] Firas Shawkat Hamid: GSM Modeling and Simulation for Urban Area. Tikrit Journal of Pure Science ISSN: 18131662 Year: 2010 Volume: 15 Issue: 1 Pages: 126-136.

[3] Parkinson, Bradford, and Spilker, Jr., James.: Global Positioning System: Theory and Applications. Vol. I, 1996.

[4] Tsui Y. and J. Bao: Fundamentals of Global Positioning System Receivers: A Software Approach. John Wiley \& Sons Inc., NewYork, NY, 2000.

[5] Sophia Y. Zheng: Signal Acquisition And Tracking For A Software GPS Receiver., Master OF Science In Electrical Engineering., Chairman., 2005.

[6] Sophia Y. Zheng: Signal Acquisition And Tracking For A Software GPS Receiver., Master OF Science In Electrical Engineering., Chairman., 2005. 
[7] N. Chadil, A. Russameesawang, and P. Keeratiwintakorn: Real-time tracking management system using GPS, GPRS and Google earth. In Proceedings of the 5th International Conference on Electrical Engineering/Electronics, Computer, Telecommunications and Information Technology, vol. 1, 2008, pp. 14-17.

[8] Francois Peyret, David Betaille, and Gaetan Hintzy: Highprecision application of GPS in the field of real-time equipment positioning. Automation in Construction, vol. 9, pp. 299-314, 2000. Elsevier.

[9] Stoleru, R.; Tian He; Stankovic, J.A.: Walking GPS: a practical solution for localization in manually deployed wireless sensor networks. Local Computer Networks, 2004. 29th Annual IEEE International Conference on, vol., no.pp. 480- 489, 16-18 Nov. 2004.

[10] K. Michael, A. McNamee, and M. Michael.: The emerging ethics of humancentric GPS tracking and monitoring. In Proceedings of the International Conference on Mobile Business, June 2006, pp. 34 43.

[11] Firas Shawkat Hsmid: The difference between IEEE 802.16 / WiMAX and IEEE 802.11/ Wi-Fi networks for Telemedicine Applications. International Journal of Recent Technology and Engineering (IJRTE), ISSN: 2277-3878, Volume-2, Issue-5, 2013.

[12] B. Hoh et al.: Preserving privacy in GPS traces via uncertainty-aware path cloaking. In CCS '07: Proceedings of the 14th ACM conference on Computer and communications security. ACM, 2007, pp. 161-171.

[13] M. Spirito: On the accuracy of cellular mobile station location estimation. IEEE Transactions on Vehicular Technology, vol. 50, no. 3, pp. 674-685, 2001. 